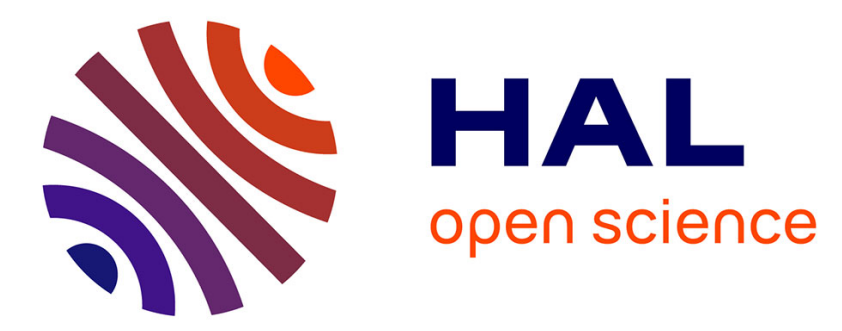

\title{
Ultrafast Atomic Diffusion Paths in Fine-Grained Nickel Obtained by Spark Plasma Sintering
}

\author{
Lucía García de La Cruz, Bernadette Domengès, Sergiy Divinski, Gerhard \\ Wilde, Eric Hug
}

\section{- To cite this version:}

Lucía García de La Cruz, Bernadette Domengès, Sergiy Divinski, Gerhard Wilde, Eric Hug. Ultrafast Atomic Diffusion Paths in Fine-Grained Nickel Obtained by Spark Plasma Sintering. Metallurgical and Materials Transactions A, 2020, 51 (7), pp.3425-3434. 10.1007/s11661-020-05791-4 . hal-03093908

\section{HAL Id: hal-03093908 https://hal.science/hal-03093908}

Submitted on 4 Jan 2021

HAL is a multi-disciplinary open access archive for the deposit and dissemination of scientific research documents, whether they are published or not. The documents may come from teaching and research institutions in France or abroad, or from public or private research centers.
L'archive ouverte pluridisciplinaire HAL, est destinée au dépôt et à la diffusion de documents scientifiques de niveau recherche, publiés ou non, émanant des établissements d'enseignement et de recherche français ou étrangers, des laboratoires publics ou privés. 


\title{
Ultrafast atomic diffusion paths in fine-grained nickel obtained by Spark
}

\section{Plasma Sintering}

Lucía García de la Cruz ${ }^{\mathrm{a}}$, Bernadette Domenges ${ }^{\mathrm{a}}$, Sergiy V. Divinski ${ }^{\mathrm{b}}$, Gerhard Wilde ${ }^{\mathrm{b}}$, Eric $\operatorname{Hug}^{\mathrm{a}^{*}}$

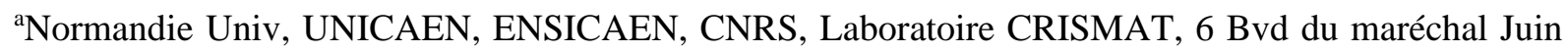
14050 Caen, France

${ }^{\mathrm{b}}$ Institute of Materials Physics, University of Münster, Wilhelm-Klemm-Str. 10, 48149 Münster, Germany

* Corresponding author

\begin{abstract}
Short-circuit diffusion in fine-grained Ni samples processed by Spark Plasma sintering has been investigated by the radiotracer technique. Ni grain boundary self-diffusion is measured in samples sintered from commercial as-received powder and from a powder processed by mechanical milling (MM). Both samples displayed high penetration of the radiotracer and ultrafast diffusion rates, which exceed the diffusivity along general high-angle grain boundaries as they are present in pure polycrystalline $\mathrm{Ni}$. A distinct profile was observed for each sample, dependent on the precursor powder. A stable penetration profile after pre-annealing at $773 \mathrm{~K}$ was observed when using commercial powder whereas a decrease in the grain boundary diffusion coefficient was depicted for the sample prepared from MM powders. The latter observation was interpreted in terms of partial relaxation of non-equilibrium grain boundaries generated by MM. Sample preparation by focused ion beam enabled the observation of interconnected porous paths in the sample prepared from commercial powders, which represent the main ultrafast diffusion path. A measurement of the surface diffusion coefficient through the pores was attempted considering a B - C type kinetic regime. The isolated pores observed in the sample prepared from MM powder, suggest a complex hierarchy of diffusion paths.
\end{abstract}

Keywords: nickel, spark plasma sintering, fine-grained microstructure, ultrafast diffusion paths, porosity structures, tracer diffusion. 


\section{Introduction}

Powder metallurgy represents a growing industry where complex shapes of a large range of materials (pure metals and alloys) can be processed in a short time and with excellent production value ${ }^{[1-3]}$. It is highly used in different domains such as aerospace electronics, nuclear energy industry, and technologies for medical or dental use and especially in the automobile industry ${ }^{[4-6]}$. Different sintering techniques enable the fabrication of custom-made microstructures including ultrafine grained (UFG), bimodal or harmonic structures ${ }^{[7-9]}$. Such microstructures are produced using powders of different characteristics. A practical tool to modify the morphology, size, chemical nature and microstructure of powders is mechanical milling (MM). Mechanical milling is a severe plastic deformation (SPD) technique where high strain is achieved from the collisions between the milling media and the powder. Adjusting the different milling parameters ${ }^{[10]}$, powders with the required characteristics can be obtained.

In the last decade, excellent mechanical properties of the above mentioned microstructures have been highlighted ${ }^{[11-14]}$ but studies concerning self-diffusion behavior are lacking. In most cases, the custom-made structures are prepared from MM powders that display UFG microstructures ${ }^{[15]}$. SPD-processed metals are known to display enhanced diffusivity due to the formation of the so-called "non-equilibrium" or "deformation modified" grain boundaries (GBs) that are in a high-energy state ${ }^{[16-18]}$. They are generated by plastic deformation and display an excessive density of extrinsic grain boundary dislocations ${ }^{[19,20]}$. This is the case for nickel samples prepared by equal channel angular pressing (ECAP) ${ }^{[21]}$ and high pressure torsion (HPT) ${ }^{[22]}$. Studies on nickel, as a model material, are very convenient as a large body of literature can be used to compare the measured results. The SPD nature of MM makes it pertinent to think that MM could also entail the formation of deformation modified $\mathrm{GBs}^{[23]}$. In fact, $\mathrm{MM}$ has been used to enhance hydrogenation of $\mathrm{Mg}$ alloys ${ }^{[24,25]}$, providing similar results as the ECAP processed samples ${ }^{[26]}$. Relaxation of deformation modified GBs can be achieved at elevated temperatures ${ }^{[27,28]}$. Nevertheless, if fast sintering techniques such as Spark Plasma Sintering (SPS) are used for compaction, the short sintering time could lead to an incomplete relaxation of deformation modified GBs leading to ultrafast diffusion paths in samples processed from MM powders. 
Previous studies concerning a Fe-40wt.\% Ni alloy processed by powder metallurgy (without application of ball milling step that insured a high purity of the final product) ${ }^{[29]}$ reported a bimodal (hierarchically ordered) distribution of internal interfaces, where interagglomerate interfaces displayed higher diffusivity than relaxed high angle grain boundaries. It is important that a similar hierarchy has been reported for SPD processed metals, too ${ }^{[30]}$. Generally, some drawbacks of powder metallurgy compared to SPD techniques include a higher level of contamination as well as the volume fraction of the residual porosity, which can highly influence diffusion properties. The number and hierarchy of diffusion paths can thus be complex in samples processed by powder metallurgy, making necessary experimental studies to elucidate diffusion processes in such systems. In the present work, nickel powders as-received and after $\mathrm{MM}$ were used to produce fine-grained microstructures after consolidation by SPS. Then, self-diffusion was investigated to explore the existence of ultrafast diffusion paths using the radiotracer method. The experimental conditions were chosen to highlight the ultrafast channels (if present) and hinder diffusion along relaxed high angle GBs.

\section{Experimental procedure}

\section{$\underline{\text { 2.1 Sample synthesis and characterization }}$}

High purity nickel powder (> $99.8 \%$ ) was used as starting material, with Fe 100 ppm, C 200 - 600 ppm, O 1000 ppm and S 10 ppm as the main impurities. The as-received powder was modified by high-energy ball milling in a Fritsh planetary pulverisette 7 with WC vials (80 $\mathrm{mL})$ and grinding balls (5 $\mathrm{mm}$ in diameter). Ball to powder ratio was fixed to 10:1, rotation speed to $300 \mathrm{rpm}$ and milling time to three hours to produce a highly deformed UFG powder. Vials were filled with high purity argon to avoid oxidation of the powders and 66.7 wt.\% anhydrous methanol was used as process control agent. Spark Plasma Sintering with a FCT System GmbH, HD25 SPS apparatus, was used for consolidation of the powders. Sintering was performed under medium dynamic vacuum ( $\sim .5 \mathrm{mbar})$ at a heating rate of 100 $\mathrm{K} / \mathrm{min}$ by continuous current and a maximum temperature of $1023 \mathrm{~K}$. The heating cycle was accompanied by a uniaxial pressure cycle with a maximum value of $75 \mathrm{MPa}$. Maximum pressure and temperature were maintained for $10 \mathrm{~min}$ to ensure good density. Disc-like specimens were obtained with a diameter of $10 \mathrm{~mm}$ and a thickness of $2.5 \mathrm{~mm}$. The density of 
the bulk samples was determined using an immersion densitometry method (mass accuracy 1 $\mathrm{mg})$.

The microstructure of the sintered samples was characterized by means of electron backscatter diffraction (EBSD) using the ESPRIT 2.0 (Bruker) software for acquisition and analysis. A step size ensuring more than 4 points per grain along a given direction was chosen for each sample. The average grain size was estimated and grain boundary character distribution (GBCD) evaluated using the following criteria. A misorientation angle of $2^{\circ}-15^{\circ}$ was set ${ }^{[31]}$ to define low angle grain boundaries (LAGBs), and Brandon's criterion ${ }^{[32]}$ to determine coincidence site lattice (CSL) fractions, calculated on a length basis. To eliminate artefacts a basic clean-up was performed before each analysis. Samples were mechanically polished and electropolished using a solution of perchloric acid, 2-butoxyethanol and ethanol at a voltage of $24 \mathrm{~V}$, to ensure an optimal surface condition. In addition, a characterization by scanning electron microscopy (SEM) combined with focused ion beam (FIB) was performed using a Dual-Beam system (FEI-HELIOS 600, Elstar Field Emission Scanning Electron Microscope column and Tomahawk Focused Ga Ion Beam column) to expose the porosity structures of the samples, through slice-and-view procedure. This means that subsequent cross-sections, $30 \mu \mathrm{m}$ - wide x $15 \mu \mathrm{m}$-deep, spaced of $\sim 70 \mathrm{~nm}$ were performed with $\mathrm{Ga}^{+}$ion beam, and SEM images were registered after each milling step, representing thus a reconstructed volume of about $30 \mu \mathrm{m}$ (length) x $15 \mu \mathrm{m}$ (depth) x $2 \mu \mathrm{m}$ (width).

\section{$\underline{2.2 \text { Radiotracer experiments }}$}

The radiotracer ${ }^{63} \mathrm{Ni}$ (half-life 100 years) was chosen to investigate self-diffusion in the sintered samples. It was available as a chloride solution and was diluted with doubledistilled water to obtain a specific activity of $\sim 5 \mathrm{kBq} / \mu \mathrm{L}$. About $2 \mu \mathrm{L}$ of the liquid tracer solution was dropped on the surface of the samples, which was mechanically polished to mirror-like quality finishing with colloidal silica (OP-S $0.04 \mu \mathrm{m})$. Once the solution was dry, the samples covered in Ta foil were sealed in evacuated silica tubes re-filled by high purity Ar $(4 \mathrm{~N} 8)$.

The temperature of the furnace was measured with a $\mathrm{Ni}-\mathrm{NiCr}$ thermocouple with an uncertainty of $\pm 1 \mathrm{~K}$. After the diffusion anneals the diameter of the samples was reduced by $\sim 0.5 \mathrm{~mm}$ to remove the effects of surface and radial diffusion. The penetration profiles were 
determined using a precision parallel grinder to remove consequently thin layers from the sample surface. The thickness of each section was calculated by measuring the mass of the sample before and after each grinding step in a microbalance (mass accuracy $0.0001 \mathrm{mg}$ ). A liquid scintillation counter (LSC), TRI-CARB 2910 TR PACKARD, Canberra Co, was used to determine the activity of each section. The counting time was set to 2 hours to have a statistical error of the counting rate of less than two counts per minute.

Penetration profiles are built as relative specific activity (proportional to the tracer concentration) plotted against the depth, enabling the determination of diffusion coefficients. The relative specific activity is obtained as a ratio between the background-corrected counting rate in the isotope-specific energy window of $2-68 \mathrm{kV}$ (in Bq) and the mass of each section. In this study, four annealing states are considered, see Table 1.

Table 1. Characteristics of each annealing state. In bold, states corresponding to diffusion annealing.

State Condition

\section{E1}

E2

E3

E4

\section{Initial state after sintering}

$400 \mathrm{~K}$ for 3 days

$\mathrm{E} 2+773 \mathrm{~K}$ overnight

$\mathrm{E} 3+400 \mathrm{~K}$ for 3 days

\section{Results}

The presence of ultrafast diffusion paths in SPS processed samples was investigated for two states with fine-grained microstructures. Sample A (Fig. 1(a)), with a grain size of 3.2 $\pm 1.8 \mu \mathrm{m}$ was produced using the as-received commercial powder as the precursor powder. Sample B (Fig. 1(b)), which exhibits an average grain size of $1.69 \pm 1.25 \mu \mathrm{m}$, was sintered from the powder obtained by MM. Despite the similar average grain size, a difference in grain size distribution is prominent (Fig. 1(c)). A log-normal distribution centered at $4 \mu \mathrm{m}$ is seen for sample A, whereas sample B displays a high fraction of smaller grains that the fraction decreases rapidly with increasing grain size. In the initial state (E1) a fraction of LAGBs is 
below $3 \%$ for both samples. The measured relative densities, $96 \%$ and $97.8 \%$ for samples A and B respectively, agree with the values reported by for SPS sintered pure Ni prepared with similar conditions ${ }^{[33]}$.
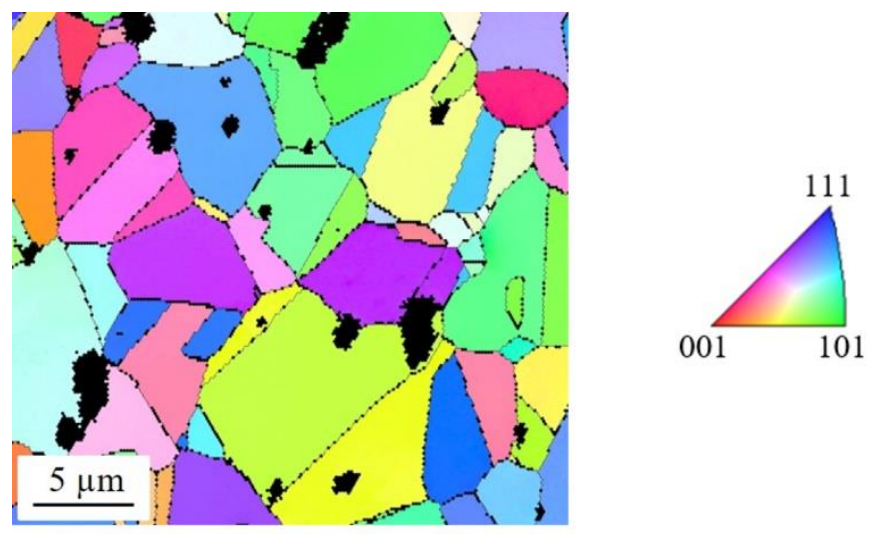

(a)
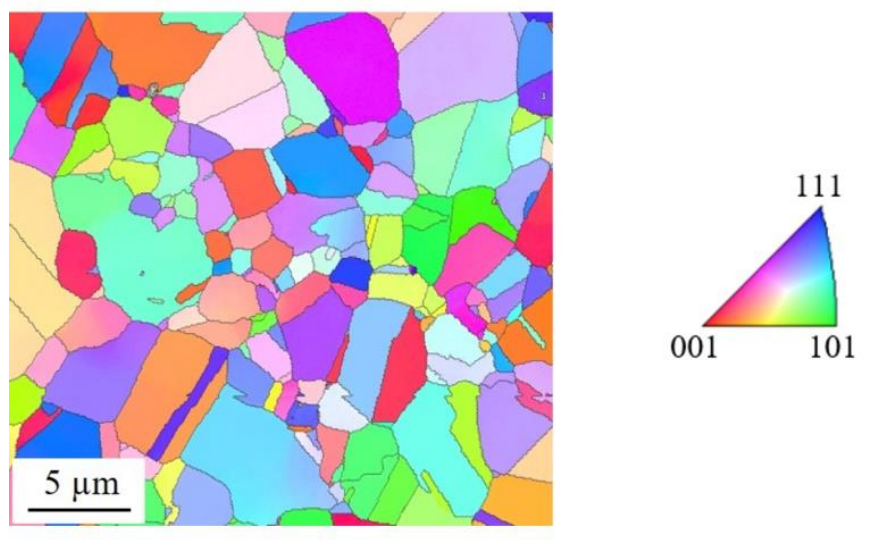

(b)

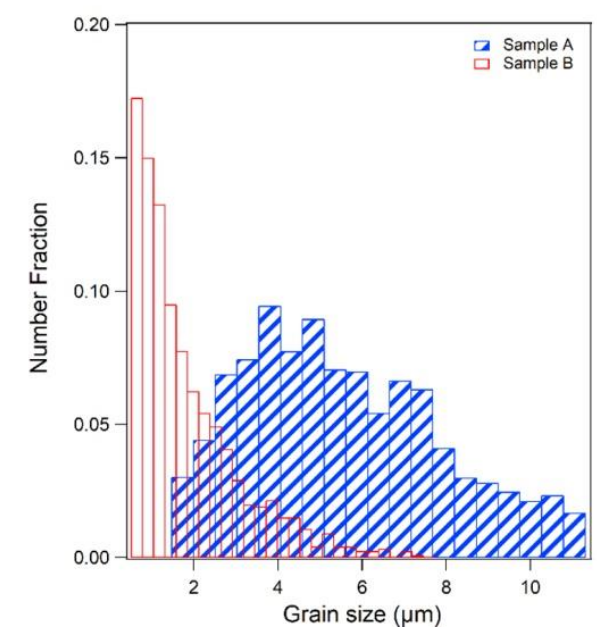

(c)

Fig. 1. EBSD orientation maps of sample A (a), sample B (b) and corresponding grain size distribution plots for both samples (c). Black spots in orientation maps correspond to badly indexed zones coinciding with pores, which are enlarged by electropolishing. 
Diffusion annealing conditions were chosen to fulfill the C-type kinetic regime of GB diffusion. According to Harrison's classification of kinetic regimes ${ }^{[34]}$, C-type kinetics dominate diffusion when the grain boundary diffusion parameter, $\alpha$ (Eq. (1)) is formally much higher than unity $(\alpha \gg 1)$.

$$
\alpha=\frac{s \delta}{2 \sqrt{D_{v} t}}
$$

Here, $s$ denotes the segregation coefficient, which equals to one for self-diffusion, $\delta$ is the GB width, measured as $0.5 \mathrm{~nm}$ for a number of FCC metals including $\mathrm{Ni}^{[35]}, D_{v}$ is the lattice diffusion coefficient and $t$ is diffusion annealing time. The parameter $\alpha$ offers an inverse measure of the leakage of diffusing atoms from GBs into the bulk ${ }^{[36]}$. A high $\alpha$ value entails negligible leaking into the bulk and $\alpha>1$ is already a good estimate of the C-type conditions for a typical radiotracer experiments ${ }^{[36]}$. Given the diffusion annealing conditions in this study, $400 \mathrm{~K}$ for three days, $\alpha$ can be determined from Eq. (1). To calculate the volume self-diffusion coefficient in nickel, the data measured by Maier et al. ${ }^{[37]}$ were used:

$$
D_{v}=9.2 \times 10^{-5} \exp \left(-\frac{278 \mathrm{~kJ} / \mathrm{mol}}{R T}\right)
$$

Here, $R$ is the gas constant. The aforementioned conditions imply the value of the grain boundary diffusion parameter $\alpha=6 \times 10^{6}$. Once the conditions for a C-type kinetic regime are fulfilled, the GB diffusion coefficient $D_{g b}$ can be determined as:

$$
D_{g b}=\frac{1}{4 t}\left(-\frac{\partial \ln \bar{c}}{\partial y^{2}}\right)^{-1}
$$

where $y$ is the penetration depth and $\bar{c}$ is the tracer concentration.

\section{$\underline{3.1 \text { Sample A }}$}

The penetration profile for sample A is presented in Fig. 2(a) after annealing at $400 \mathrm{~K}$ for three days (state E2). Large penetration depths are seen, $y>25 \mu \mathrm{m}$, which indicates the presence of fast short-circuit diffusion paths. At low temperature, the conventional diffusion profile displays a sharp decrease in the near surface concentration (probably due to some tracer evaporation) followed by deep penetration of the tracer. Thus, the unexpected shape of the concentration profile suggests a complex structure composed of several kinds of shortcircuit diffusion paths. Nevertheless, given the high value of $\alpha$, the Gaussian-type solution 
(Eq. (3)) of the diffusion problem can be adopted as a first approach to analyze the penetration profile by plotting relative specific activity against $y^{2}$ (Fig. 2(b)). The diffusion profile can be divided in two nearly linear parts, which represent grain boundary diffusivities of $D_{g b}{ }^{1}=$ $6.04 \times 10^{-17} \mathrm{~m}^{2} / \mathrm{s}$ and $D_{g b^{2}}=1.40 \times 10^{-16} \mathrm{~m}^{2} / \mathrm{s}$, respectively. Short-circuit diffusion in this sample proceeds faster than along relaxed high angle GBs in high-purity $\mathrm{Ni}\left(D_{g b}^{r e l}\right)$ at $400 \mathrm{~K}$ by orders of magnitude ${ }^{[35]}, D_{g b}^{r e l}=1.08 \times 10^{-25} \mathrm{~m}^{2} / \mathrm{s}$.

Furthermore, diffusivity in the first part $\mathrm{Dgb}^{1}$ is comparable to the values measured on ECAP processed $\mathrm{Ni}$, which exhibits deformation-modified $\mathrm{GBs}^{[16]}$. A hypothetical impurity effect related to contamination during the synthesis of the samples can be discarded as in FCC metals GB diffusivity decreases with increasing impurity levels ${ }^{[38]}$. A presence of Bi atoms which could induce pre-melting grain boundary phase transition and enhance GB diffusion as in the $\mathrm{Cu}$-Bi system ${ }^{[39]}$ could be safely disregarded. Moreover, such effects would be expected at higher temperatures of about 0.8 of the melting point ${ }^{[39]}$.

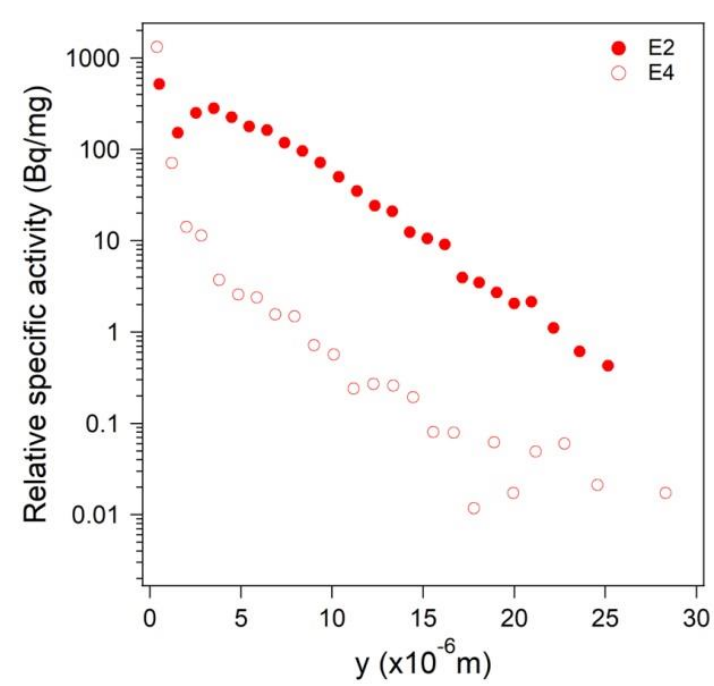

(a)

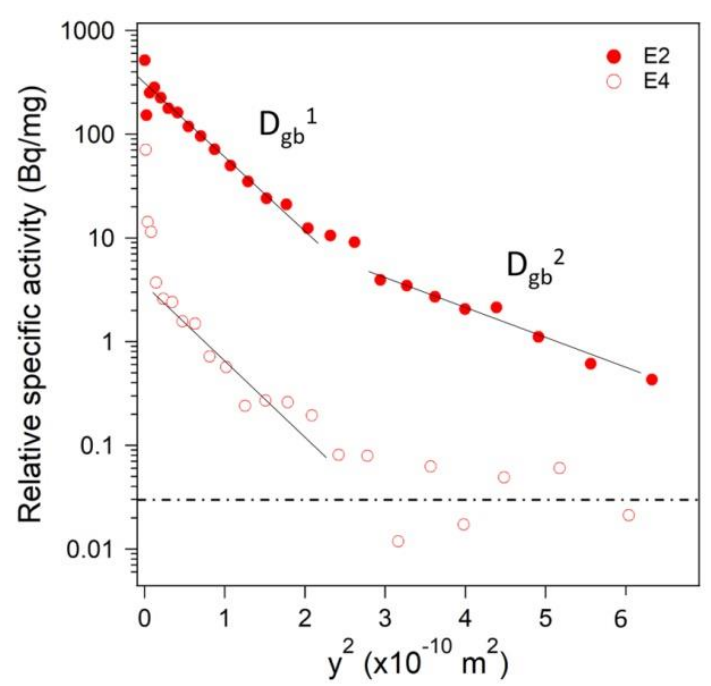

(b)

Fig. 2. Penetration profiles plotted against y (a) and $\mathrm{y}^{2}$ (b) for diffusion annealing at $400 \mathrm{~K}$ for 3 days of ${ }^{63} \mathrm{Ni}$ in SPS Ni produced from commercial as-received powders (sample A). The filled symbols represent the profiles obtained after the first diffusion annealing (E2) and the open symbols after the second diffusion annealing (E4). Background level indicated by dashed line. 
Although deformation modified GBs are not expected in this specimen (processed from commercial powder), the applied pressure and non-equilibrium conditions originating from electric current in the sintering process (rapid heating and cooling) could create "nonequilibrium" GBs, from the thermomechanical treatment. To explore this possibility, a preannealing treatment (E3) was performed to the same sample followed by a second diffusion experiment (E4) with the same conditions of temperature and time as E2. The measured penetration profile can be observed in Fig. 2(a) (E4).

Unlike for state E2, a single slope is depicted for state E4 (Fig. 2(b)) displaying a GB diffusion coefficient of $D_{g b}=6.13 \times 10^{-17} \mathrm{~m}^{2} / \mathrm{s}$, which is very close to $D_{g b}{ }^{1}$ measured in the annealing state E2. Hence, the same short-circuits can be accountable for diffusion in both states and no evolution of diffusive paths is observed after pre-annealing at $773 \mathrm{~K}$ (E3). The main difference between the profiles is a shift of the curve towards lower values of isotope concentration, which is presumably related to sample preparation. Due to the radioactive nature of the samples after the first diffusion annealing (E2), surface preparation must be performed in the parallel grinder for the second diffusion experiment. This could lead to slightly more uneven surface that can reflect on the absolute value of the measured activity. However, these features may induce some artefacts only at small depths below several microns and these points are not included in the present analysis (Fig. 2).

\section{$\underline{3.2 \text { Sample B }}$}

In sample $\mathrm{B}, \mathrm{GB}$ diffusion was also investigated considering the $\mathrm{C}$-type kinetic regime using the same annealing conditions as for sample A. The corresponding concentration profile is plotted in Fig. 3(a) (E2). The penetration profile exhibits again two distinct sections with a steep and a flat slope. This type of profile which displays a "slow" and a "fast" branch has been reported for SPD-processed $\mathrm{Cu}-\mathrm{Zr}^{[30]}, \mathrm{Cu}-\mathrm{Pb}^{[40]}$ and $\mathrm{Ni}^{[21]}$ and for powder metallurgy produced $\mathrm{Fe}-\mathrm{Ni}$ alloy ${ }^{[29]}$ as a result of a hierarchy of diffusion paths in UFG specimens. An analysis of the concentration profile plotted against $y^{2}$ (Fig. 3(b)) provides a GB diffusion coefficient of $D_{g b}{ }^{l}=3.01 \times 10^{-17} \mathrm{~m}^{2} / \mathrm{s}$ and $D_{g b}{ }^{2}=3.33 \times 10^{-16} \mathrm{~m}^{2} / \mathrm{s}$, respectively for the slow and fast branch. In fact, these values are very similar to those obtained for sample A.

A pre-annealing treatment (E3) followed by diffusion annealing (E4) was performed to explore the presence of remnant deformation induced GBs in sample B. The measured 
penetration profile can be observed in Fig. 3(a) (E4). A single branch is observed of stepper slope than the one observed for E2. When plotting concentration as a function of $y^{2}$ (Fig. 3(b) (E4)) a GB diffusion coefficient of $D_{g b}=1.84 \times 10^{-17} \mathrm{~m}^{2} / \mathrm{s}$, is obtained. Contrary to sample A, the annealing treatment (E3) performed in this sample triggered a moderate decrease in the GB diffusion coefficient.

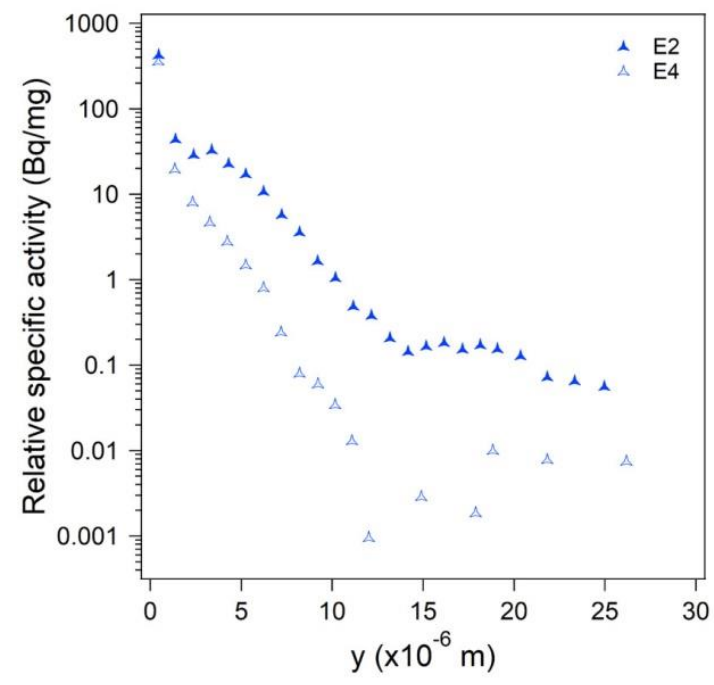

(a)

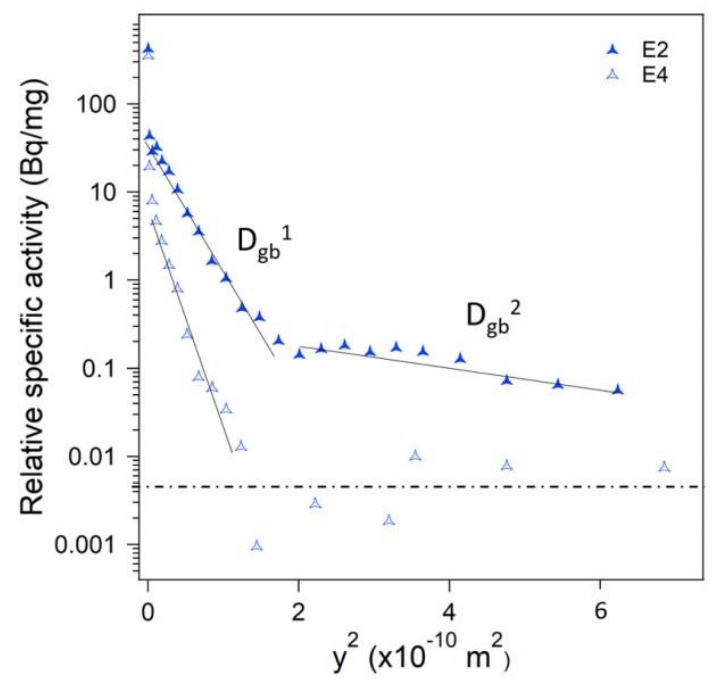

(b)

Fig. 3. Penetration profiles plotted against $y$ (a) and $y^{2}$ (b) for diffusion annealing at $400 \mathrm{~K}$ for 3 days of ${ }^{63} \mathrm{Ni}$ in Ni produced from MM powders (sample B). The filled symbols represent the profiles obtained after the first diffusion annealing (E2) and the open symbols after the second diffusion annealing (E4). Background level indicated by dashed line.

To elucidate the possible causes of the reduced diffusion rate in $\mathrm{E} 4$, the microstructure was examined for each annealing state and Table 2 collects the measured results. No remarkable changes in grain size or GBCD can be observed besides statistical dispersion. Hence, the evolution of the penetration profile hints towards an intricate structure of ultra-fast diffusion paths that would include deformation modified GBs. The relaxation upon annealing of a fraction of such boundaries would thus result in the reduced effective diffusivity. 
Table 2. Microstructural characteristics of sample B for different annealing states.

\begin{tabular}{cccccc}
\hline State & Grain size $(\mu \mathrm{m})$ & HAB & $\sum 3$ & Other CSL & LAGB \\
\hline E1 & $1.69 \pm 1.25$ & 0.474 & 0.450 & 0.050 & 0.026 \\
E2 & $1.56 \pm 1.19$ & 0.457 & 0.470 & 0.048 & 0.021 \\
E3 & $1.75 \pm 1.31$ & 0.446 & 0.492 & 0.046 & 0.016 \\
E4 & $1.92 \pm 1.44$ & 0.443 & 0.489 & 0.044 & 0.024 \\
\hline
\end{tabular}

\section{Discussion}

Diffusion experiments were performed on samples A and B at low annealing temperature $(400 \mathrm{~K})$ showing distinct concentration profiles. In sample A, processed from commercial powder, the experimental penetration profile displayed an unexpected deep penetration of the radiotracer that persisted after annealing at higher temperature $(773 \mathrm{~K})$ (Fig. 2(a)). The high transport rates in this sample are closer in value to ECAP processed Ni than to coarse-grained Ni. Interestingly, Divinski et al. ${ }^{[16]}$ reported an increase in diffusion rates in ECAP Ni, after a pre-annealing under certain conditions. In fact, residual free volume and vacancy-like defects characteristic of the relaxation of non-equilibrium GBs evolved during pre-annealing to form percolating porosity. Thus, considering the high diffusivity rate and the stable concentration profile after annealing, the ultra-fast diffusion observed in sample A could be due to interconnected porosities. To explore this hypothesis, the slice-and-view study of the initial state (E1) of sample A was performed in the Dual-Beam. This tomographic reconstruction enables the inspection of possible connections between pores as well as the determination of the geometry of such structures. The resulting images are displayed in Fig. 4(a-c) at three subsequent milling steps. Big size pores can be observed mostly decorating grain boundaries. In Fig. 4(a) two big porosities situated close to each other (indicated by the black arrow), result interconnected when inspected after further milling (Fig. 4(b)) creating intricate geometries. This example is not isolated but generalized for this sample (Fig. 4(c)). 


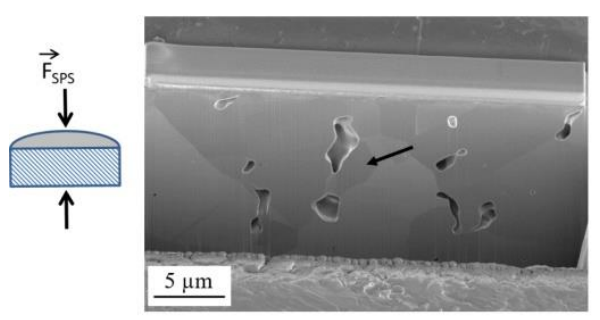

(a)

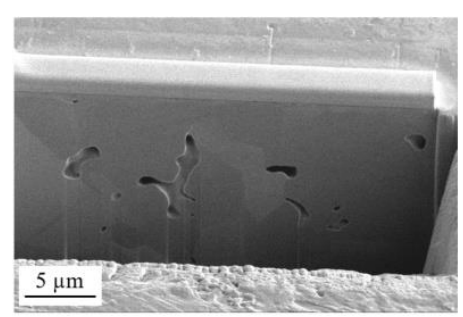

(b)

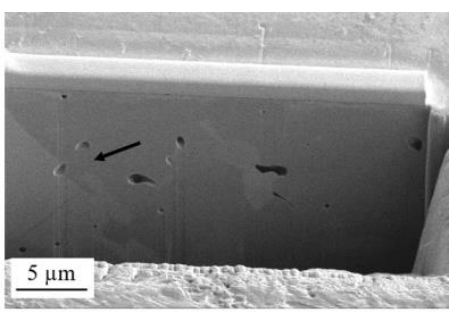

(c)

Fig. 4.SEM images of sample A in state E1 after preparation by FIB at three consecutive depths (a), (b) and (c). Arrows indicate two pores that become interconnected.

The coherence in the concentration profiles measured in states E2 and E4 suggests no relaxation of "non-equilibrium" GBs. Thus, the observations of the porosity structures seem to indicate a combination of diffusion paths consisting of a network of porous channels and random HABs. The high linearity displayed by the diffusion profile of sample A referred to penetration depth (Fig. 2(a)) suggests a "modified" B-type kinetic regime, where the diffusion profile becomes linear against $y^{6 / 5}$. GB diffusion in the B-type regime is accompanied by volume diffusion from leaking, where volume diffusion fluxes of neighboring grains do not overlap. In the case of sample A, the diffusing system would be composed of porous channels (Fig. 4(a)) as the principal high diffusivity path and leakage would occur into relaxed highangle GBs. Thus, adopting a model that considers leaking at points where the porous channels meet GBs, a "surface" diffusion coefficient through the pores $\left(D_{p}\right)$ could be estimated. To this aim, the model proposed by Divinski et al. for a nanostructured $\gamma$-Fe-40wt.\%Ni alloy ${ }^{[29,41]}$, could be adapted to our system. The alloy, with a relative density of $98 \%$, displayed a microstructure with two levels of organization. At a larger scale, the microstructure exhibited polyhedral large agglomerates or clusters with porosities mostly located in the interfaces. At a smaller scale, agglomerates were composed of nanosized grains. In such system, interagglomerate boundary diffusion displayed enhanced diffusivity compared to GB diffusion in the nanosized grains. Thus, inter-agglomerate diffusion would constitute the main diffusion path leaking towards grain boundaries of the nanograins. To analyze the concentration profiles obtained al low temperature $(653 \mathrm{~K}-750 \mathrm{~K})$ they proposed a " $\mathrm{C}-\mathrm{B}$ kinetic regime" were a double product for inter-agglomerate interfaces $\left(P_{a}\right)$ can be determined as follows:

$$
P_{a}=\frac{\delta_{a} \cdot D_{a}}{\lambda_{a}}=0.661\left(\frac{4 D_{g b}}{t}\right)^{1 / 2}\left(-\frac{\partial \ln \bar{c}}{\partial y^{6 / 5}}\right)^{-5 / 3}
$$


Here, $\delta_{a}$ is the agglomerate interface width, $D_{a}$ is the diffusion coefficient of the interfaces between agglomerates and $\lambda_{a}$ is a factor added to take into account the outdiffusion to the GBs exclusively at crossing points between the inter-agglomerate interfaces and GBs. For polyhedral grains:

$$
\lambda_{a}=\frac{\pi \delta}{2 d}
$$

where $d$ represent grain size. Thus, if the grain boundary diffusion coefficient is known, using Eq. (4) and (5), $D_{a}$ can be estimated assuming an arbitrary agglomerate interface width $\delta_{a}$.

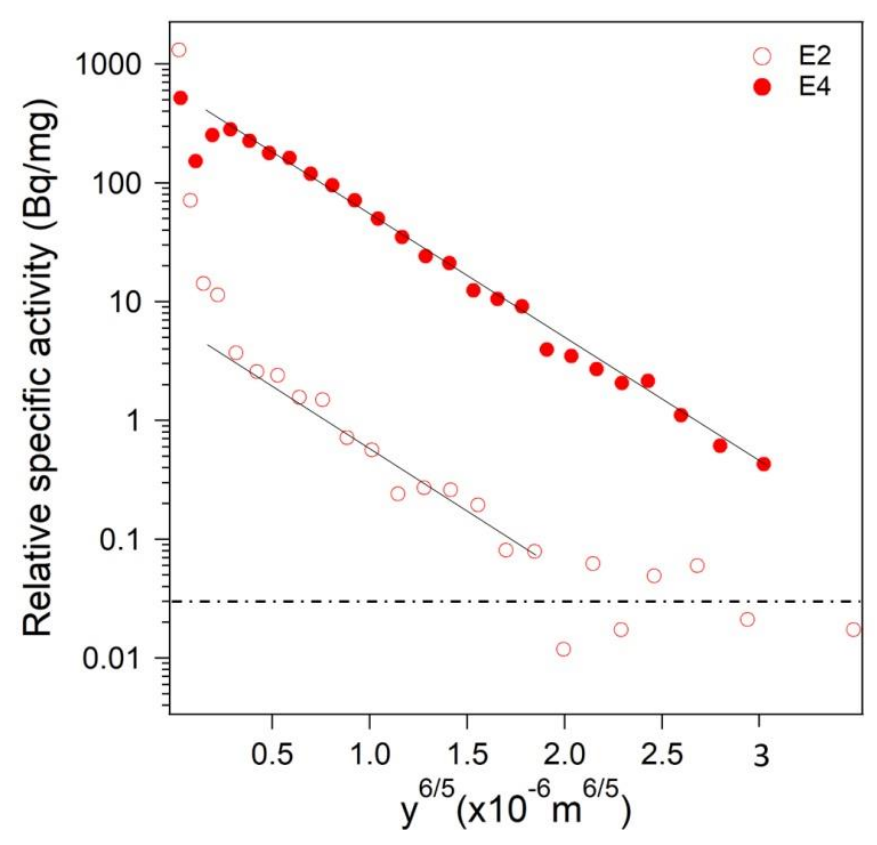

Fig. 5. Penetration profiles plotted against $y^{6 / 5}$ for diffusion annealing at $400 \mathrm{~K}$ for 3 days of ${ }^{63} \mathrm{Ni}$ in SPS Ni produced from commercial as-received powders (sample A). Background level indicated by dashed line.

In our case, considering sample $\mathrm{A}, D_{g b}$ represents the GB diffusion coefficient in relaxed HABs, thus we can assume $D_{g b}=D_{g b}^{r e l}=1.08 \times 10^{-25} \mathrm{~m}^{2} / \mathrm{s}$. If $\delta_{a} \sim 1 \mathrm{~nm}$ is assumed ${ }^{[41]}$ an approximate value of $D_{a}$ could be estimated where $D_{a}=D_{p}$. The diffusion profile of sample A plotted against $y^{6 / 5}$ is displayed in Fig. 5 for states E2 and E4. The estimated surface diffusion coefficient through porous channels determined from Eq. (4) is $D_{p}=1.40 \times 10^{-13} \mathrm{~m}^{2} / \mathrm{s}$ and $D_{p}=1.28 \times 10^{-13} \mathrm{~m}^{2} / \mathrm{s}$ respectively for states E2 and E4. These values represent a 
diffusivity three orders of magnitude higher than diffusion through non-equilibrium GBs. Considering the complexity of diffusion systems that combine such boundaries with interconnected cracks, the value of $D_{p}$ obtained in this study could be used to develop a model that takes into account the different diffusion features. Furthermore, such high values of $D_{p}$ are typical for surface diffusion rates in $\mathrm{Ni}^{[42]}$.

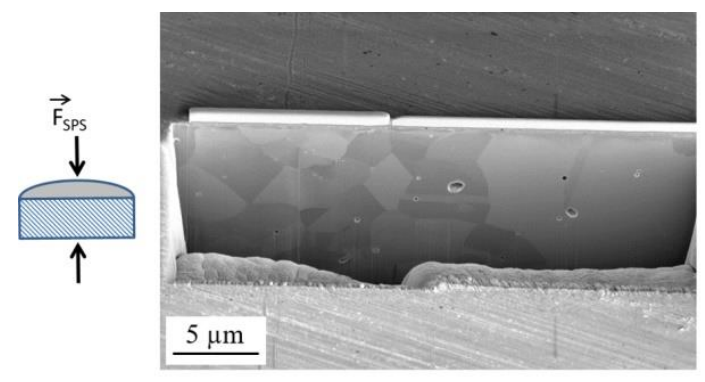

(a)

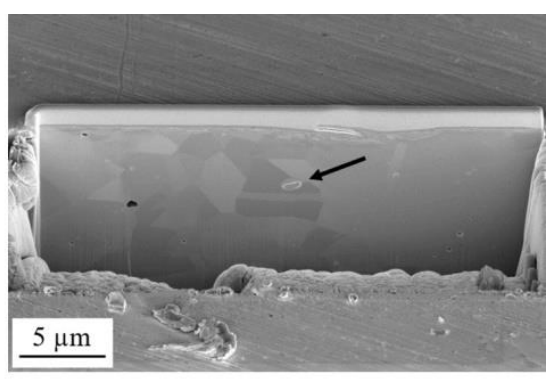

(b)

Fig. 6. SEM images of sample B in state E1 after preparation by FIB at two consecutive depths. The arrow indicates a pore visible at both depths.

In sample B, processed from MM powders, ultra-fast diffusion was observed at $400 \mathrm{~K}$, decreasing after annealing at $773 \mathrm{~K}$ overnight (Fig. 3(a)). The difference in the shape of the concentration profile between samples A and B anticipates a different hierarchy of the diffusion paths. SEM images performed at different depths (Fig. 6) display a different geometry in the pores observed in sample B, in comparison with sample A. In fact, the morphology of the precursor powders ${ }^{[43]}$ (commercial or $\mathrm{MM}$ ) has a big impact in the sintering process, which is responsible for the differences in the porosity structures between the two samples. In sample B, isolated spherical pores can be observed, located at grain boundaries as well as in the bulk of grains. The small size pores, which display in most cases a diameter below $0.5 \mu \mathrm{m}$, disappear after milling $\sim 0.5 \mu \mathrm{m}$ in depth with the exception of the pore indicated by the arrow in Fig. 6(b). Unlike sample A, no interconnected pores were depicted in sample B. Thus, a different model of ultrafast paths has to be conceived for a better analysis of GB diffusion for this sample. Such model must consider the isolated pores located at grain boundaries as well as the presence of remnant deformation modified grain boundaries from the MM process. 
Different atomic diffusion paths thus control diffusivity in samples A and B. A stable structure including ultrafast diffusion through a network of interconnected pores is depicted for sample A, contrary to the unstable structure that includes isolated pores and nonequilibrium GBs in sample B.

\section{Summary}

The present study has uncovered ultrafast diffusion paths on Ni samples processed by powder metallurgy. A clear difference in the diffusion behavior has been reported, which depends on the powder used for the synthesis of the specimens. The sintering process using commercial powders or MM powders induces pores of different geometry, irregular in the first case and spherical in the second case. Likewise, an interconnected network of pores is observed in sample A contrary to isolated pores in sample B. Similarly to SPD processed metals, which develop percolating channels during the deformation process or after certain annealing treatments ${ }^{[44]}$, the porous network in sample A provides a long-range atomic transport. On the other hand, the lack of non-equilibrium GBs simplifies the analysis of the penetration profile of sample A, where a modified B-type kinetic regime can be considered. An approximate value for surface diffusivity through the porous channels was obtained and could serve as starting point to develop a model that takes into account interconnected pores as ultra-fast diffusion paths.

The isolated pores observed in sample B hinder direct diffusion through porosities, which reflected in the concentration profile. It showed good linearity when considering the Ctype kinetic regime and a decrease in diffusivity was observed after pre-annealing at $773 \mathrm{~K}$. Such change in the diffusion profile suggests the presence of remnant deformation modified GBs, created during MM of the powder. A more complex hierarchy of ultrafast diffusion paths including such GBs and the isolated pores constitutes the diffusion system for this sample and need to be studied in more depth.

In addition to the precursor powder, sintering parameters such as temperature and pressure can be tuned to produce samples with the desired porosity fraction. High diffusivity can be detrimental when samples are submitted to aggressive environmental conditions for processes such as oxidation or corrosion. Nevertheless, it is also a requirement for surface treatments such as nitriding of steels of steel or hydrogen storage ${ }^{[44,45]}$. Thus, the above- 
mentioned results must be kept in mind when choosing the synthesis parameters to produce the desired microstructures, using powder metallurgy.

Acknowledgements. This work was performed with the financial support of the program EQUIPEX GENESIS, Agence Nationale de la Recherche (ANR-11-EQPX-0020) for DualBeam experiments. A partial financial support from the German Science Foundation (DFG) via research project is acknowledged.

\section{References}

1. J.M. Torralba, Improvement of Mechanical and Physical Properties in Powder Metallurgy,, in: S. Hashmi (Ed.) Advanced forming technologies, Elsevier, 2014, pp. 281-93.

2. W.B. James, Powder Metallurgy : Methods and Applications, in: P.K.S.a.J.W. Newkirk (Ed.) Powder Metal., ASM International, 2015, pp. 907.

3. M. Suarez, A. Fernandez, J.L. Menendez, R. Torrecillas, H.U.J. Hennicke, R. Kirchner, and T. Kessel, Challenges and Opportunities for Spark Plasma Sintering: A Key Technology for a New Generation of Materials, in: B. Ertug (Ed.) Sintering Applications, IntechOpen, 2013.

4. P. Ramakrishnan: Indian J. Hist. Sci., 1983, vol. 18, pp. 109-14.

5. D.A. Levina, L.I. Chernyshev, and N.V. Mikhailovskaya: Powder Metall. Met. Ceram., 2007, vol. 46, pp. 202-05.

6. L.A. Dobrzanski, Goals and Contemporary Position of Powder Metallurgy, in: L.A. Dobrzanski (Ed.) Powder Metallurgy. Fundamentals and Case Studies, IntechOpen, 2017, pp. 1-17.

7. T. Sekiguchi, K. Ono, H. Fujiwara, and K. Ameyama: Mater. Trans., 2010, vol. 51, pp. 39-45.

8. J. Gubicza, H.-Q. Bui, F. Fellah, and G. Dirras: J. Mater. Research, 2009, vol. 24, pp. 217-26.

9. Z. Zhang, D. Orlov, S.K. Vajpai, B. Tong, and K. Ameyama: Advanced Engineering Materials, 2015, vol. 17, pp. 791-95.

10. C. Suryanarayana: Prog. Mater. Sci., 2001, vol. 46, pp. 1-184.

11. B. Flipon, C. Keller, L.G.d.1. Cruz, E. Hug, and F. Barbe: Mater. Sci. Eng. A, 2018, vol. 729, pp. $249-56$.

12. G.D. Dutel, D. Tingaud, P. Langlois, and G. Dirras: J. Mater. Sci., 2012, vol. 47, pp. 7926-31.

13. C. Sawangrat, O. Yamaguchi, S.K. Vajpai, and K. Ameyama: Mater. Trans., 2014, vol. 55, pp. 99-105. 
14. Y. Zhao, T. Topping, J.F. Bingert, J.J. Thornton, A.M. Dangelewicz, Y. Li, W. Liu, Y. Zhu, Y. Zhou, and E.J. Lavernia: Adv. Mater., 2008, vol. 20, pp. 3028-33.

15. D.B. Bober, A. Khalajhedayati, M. Kumar, and T.J. Rupert: Metall. Mater. Trans. A, 2016, vol. 47, pp. 1389403.

16. S.V. Divinski, G. Reglitz, H. Rösner, Y. Estrin, and G. Wilde: Acta Mater., 2011, vol. 59, pp. $1974-85$.

17. S.V. Divinski: Diffus. Found., 2015, vol. 5, pp. 57-73.

18. G. Wilde, and S.V. Divinski: Mater. Trans., 2019, vol. 60, pp. 1302-15.

19. H. Miyamoto: Mater. Trans., 2016, vol. 57, pp. 559-72.

20. A. Hasnaoui, H.V. Swygenhoven, and P.M. Derlet: Acta Mater., 2002, vol. 50, pp. 3927-39.

21. S.V. Divinski, G. Reglitz, I.S. Golovin, M. Peterlechner, R. Lapovok, Y. Estrin, and G. Wilde: Acta Mater., 2015, vol. 82, pp. 11-21.

22. D. Prokoshkina, L. Klinger, A. Moros, G. Wilde, E. Rabkin, and S.V. Divinski: Acta Mater., 2014, vol. 69, pp. 314-25.

23. A.A. Nazarov, A.E. Romanov, and R.Z. Valiev: Nanostruct. Mater., 1995, vol. 6, pp. 775-78.

24. G. Liang, J. Huot, S. Boily, A.V. Neste, and R. Schulz: J. Alloy Compd, 1999, vol. 292, pp. 247-52.

25. A. Zaluska, L. Zaluski, and J.O. Ström-Olsen: J. Alloy Compd, 1999, vol. 288, pp. 217-25.

26. V.M. Skripnyuk, E. Rabkin, Y. Estrin, and R. Lapovok: Acta Mater., 2004, vol. 52, pp. 405-14.

27. S. Poulat, B. Décamps, and L. Priester: Phil. Mag. A, 1999, vol. 79, pp. 2655-80.

28. A.A. Nazarov: Interface Sci., 2000, vol. 8, pp. 315-22.

29. S.V. Divinski, F. Hisker, Y.S. Kang, J.S. Lee, and C. Herzig: Interface Sci., 2003, vol. 11, pp. 67-80.

30. Y. Amouyal, S.V. Divinski, Y. Estrin, and E. Rabkin: Acta Mater., 2007, vol. 55, pp. 5968-79.

31. V. Randle: Mater. Charact., 2001, vol. 47, pp. 411-16.

32. A.H. King, and S. Shekhar: J. Mater. Sci., 2006, vol. 41, pp. 7675-82.

33. T. Borkar, and R. Banerjee: Mater. Sci. Eng. A, 2014, vol. 618, pp. 176-81.

34. L.G. Harrison: Trans. Faraday Soc., 1961, vol. 57, pp. 1191-99.

35. S.V. Divinski, G. Reglitz, and G. Wilde: Acta Mater., 2010, vol. 58, pp. 386-95.

36. A. Paul, T. Laurila, V. Vuorinen, and S.V. Divinski, Thermodynamics, Diffusion and Kirkendall Effect in Solids, Springer International Publishing Switzerland, 2014.

37. K. Maier, H. Mehrer, E. Lessmann, and W. Schüle: Phys. Stat. Sol. B, 1976, vol. 78, pp. 689-98.

38. D. Prokoshkina, V.A. Esin, G. Wilde, and S.V. Divinski: Acta Mater., 2013, vol. 61, pp. 5188-97. 
39. S.V. Divinski, M. Lohmann, C. Herzig, B. Straumal, B. Baretzky, and W. Gust: Phys. Rev. B, 2005, vol. 71, pp. 104104.

40. J. Ribbe, D. Baither, G. Schmitz, and S.V. Divinski: Scripta Mater., 2009, vol. 61, pp. 129-32.

41. S.V. Divinski, F. Hisker, Y.S. Kang, J.S. Lee, and C. Herzig: Zeit. Metall., 2002, vol. 93, pp. 265-72.

42. R.T. Tung, and W.R. Graham: Surf. Sci., 1980, vol. 97, pp. 73-87.

43. L. García de la Cruz, B. Flipon, C. Keller, M. Martinez, and E. Hug: AIP Conf. Proc., 2017, vol. 1896, pp. 200002.

44. S.V. Divinski, J. Ribbe, G. Reglitz, Y. Estrin, and G. Wilde: J. Appl. Phys., 2009, vol. 106, pp. 063502.

45. H. Gleiter: Prog. Mater. Sci., 1989, vol. 33, pp. 223-315. 